\title{
Potentiation of Hyperthermic Effects Using Hydralazine
}

\author{
Takeo Hasegawa Ph. D. ${ }^{1)}$, Tatumi Ishiguro B. S. ${ }^{2)}$, \\ Taichi Oshima M. D. ${ }^{3)}$, and Yoshimasa Tanaka M. D. ${ }^{3)}$ \\ 1) Department of Radiological Technology. Suzuka University of Medical Science and Tech- \\ nology. Suzuka Mie 510-02. \\ 2) Department of Radiology. Daiori Medical Center of Osaka Textile Health Insurance \\ Organization. Osaka 559. \\ 3) Department of Radiology, Kansai Medical University Moriguchi city OSAKA 570.
}

\begin{abstract}
It is said that the efficacy of hyperthermia for malignancies depend on various vasophsiological factors in the tumor, such as $\mathrm{pH}$, blood flow and oxygen tension (p02). The present animal experiment was performed to ascertain how the effect of hyperthermia can be enhanced by administration of hydralazine (HYD), a vasodilator, wich is known to decrease the local blood flow selectively and accordingly, decelerate thermal diffusion in the tumor. HYD $(2-5 \mathrm{mg} / \mathrm{kg})$ was injected intraperitoneally 20 min before hyperthermia, which was done for 20-30 $\min$ at $43^{\circ} \mathrm{C}$ by the use of a thermo-static water bath. The tumor quadrupling time was 4.5 days in the untreated FM3A group, 5.4 days in the HYD treated FM3A group, 8.5 days in the heat alon FM3A group and 11.2 days in the HYD plus heat treated FM3A group. Those in the respective SCC-VII group were 7.7 days, 9.0 days, 11.1 days and 14.0 days. The enhancement ratio was 2.48 in the treatment for FM3A tumor and 1.81 in the treatment for SCC-VII tumor. Thus, these results indicate that HYD can increase the therapeutic efficiency of local hyperthermia treatment by inducing the change of microenvironment i. e. low $\mathrm{pH}$, tumor hypoxia, deficient nutrition secondary to blood flow diminution which increase the sensitivity of tumor to heat.
\end{abstract}

Key words : Hyperthermia, Blood Flow, Vasodilater, In vivo.

\section{I . Introduction}

Changes in tissue blood flow is related to thermo-diffusion due to blood circulation, tissue oxygen tension and nutritional condition. Therefore, therapeutic gain by hyperthermia and

Received 9 December 1994 
radiation therapy for malignant tumors depends largely upon these factors ${ }^{\left.12)^{3) 4}\right)}$. It is known that tumors in general have a necrotic area of the center and its periphery is in a hypoxic state because of a low blood flow ${ }^{5}$. This area is not easily accessible to anti-cancer drugs, in addition, the hypoxic condition makes those lesions tolerant to chemotherapy and radiotherapy. In this study we confirmed that the anti-tumor effect of hyperthermia was enhanced by hydralazine (HYD) as a vasodilator. HYD dilates normal blood vessels, which have smooth muscle, thereby increasing the blood flow of normal tissues. However, tumor vessels are poorly responsive to the drug because of the scarcity of local blood flow, so that the local blood flow is reduced by a steal effect following HYD administration ${ }^{6)}$. It is surmised, therefore, that HYD administration leads to the decrease of blood flow and the aggravation of the nutritional cindition, and the cytotoxicity of hyperthermia is augmented and, at the same time, the ability to repair hyperthermia induced damage.

\section{Materials and Methods}

Four week old male $\mathrm{C} 3 \mathrm{H}$ mice were used. The tumor cell lines used were FM3A and SCC-VII. These cells $\left(5 \times 10^{5}-2 \times 10^{6}\right.$ cells $\left./ 0.05 \mathrm{ml}\right)$ were transplanted into the femoral subcutis. The experiment was started 10 days after transplantation when the tumor grew to 7 $\mathrm{mm}$ in diameter. An appropriate amount of HYD to make a dose of $1 \mathrm{mg} / \mathrm{kg}, 2.5 \mathrm{mg} / \mathrm{kg}$, or $5 \mathrm{mg} / \mathrm{kg}$ was dissolved in $0.3 \mathrm{ml}$ of physiological saline and administrered into the peritoneal cavity. HYD was injected intraperitoneally $20 \mathrm{~min}$ before hyperthermia, which was done for $20-30 \mathrm{~min}$ at $43^{\circ} \mathrm{C}$ by the use of a thermo-static water bath. Tumor sizes were measured three times a week and volumetry was performed according to the formula; $\pi \mathrm{abc} / 6$, where $\mathrm{a}, \mathrm{b}$ and $\mathrm{c}$ indicate three perpendicular diameter. Time-course changes in local blood flow were monitored by use of a laser doppler flow-meter (ALF 21, Advance) and a special sensor $(500 \mu \mathrm{m}$ in outer diameter) inserted into the tumor. The tissue oxygen tension (p02) was measured by polarographic mcthod. The blood flow distribution within the tumor was studied in the following manner: Hoechst-33342, a fluorescence dye (excitation $=352 \mathrm{~nm}$,

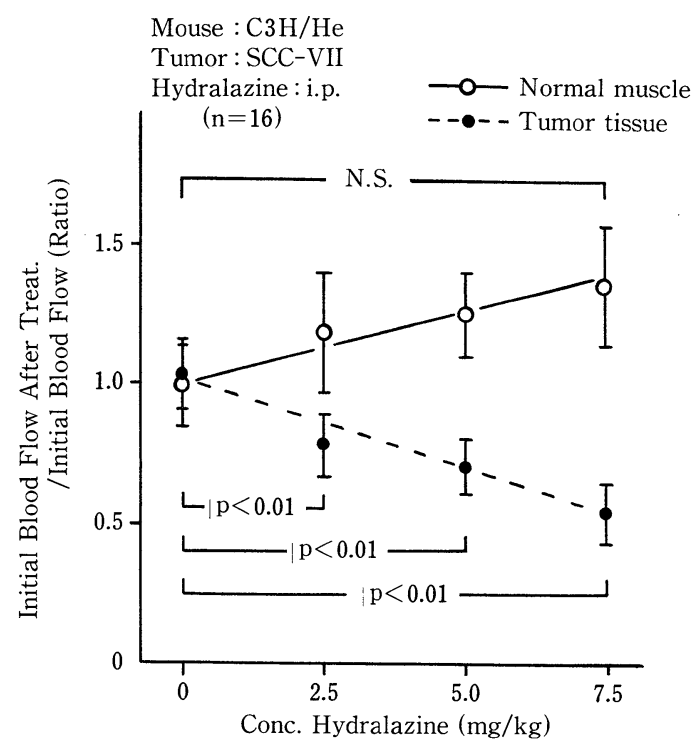

Normal tissue did not show the decrease of blood flow after HYD infusion. But tumor tissue shown the decrease of blood flow significantly.

Fig. 1 Changes in blood flow in SCC-VII tumor $(\bullet)$ and intact muscle $(O)$ after infusion of hydralazine (HYD). 


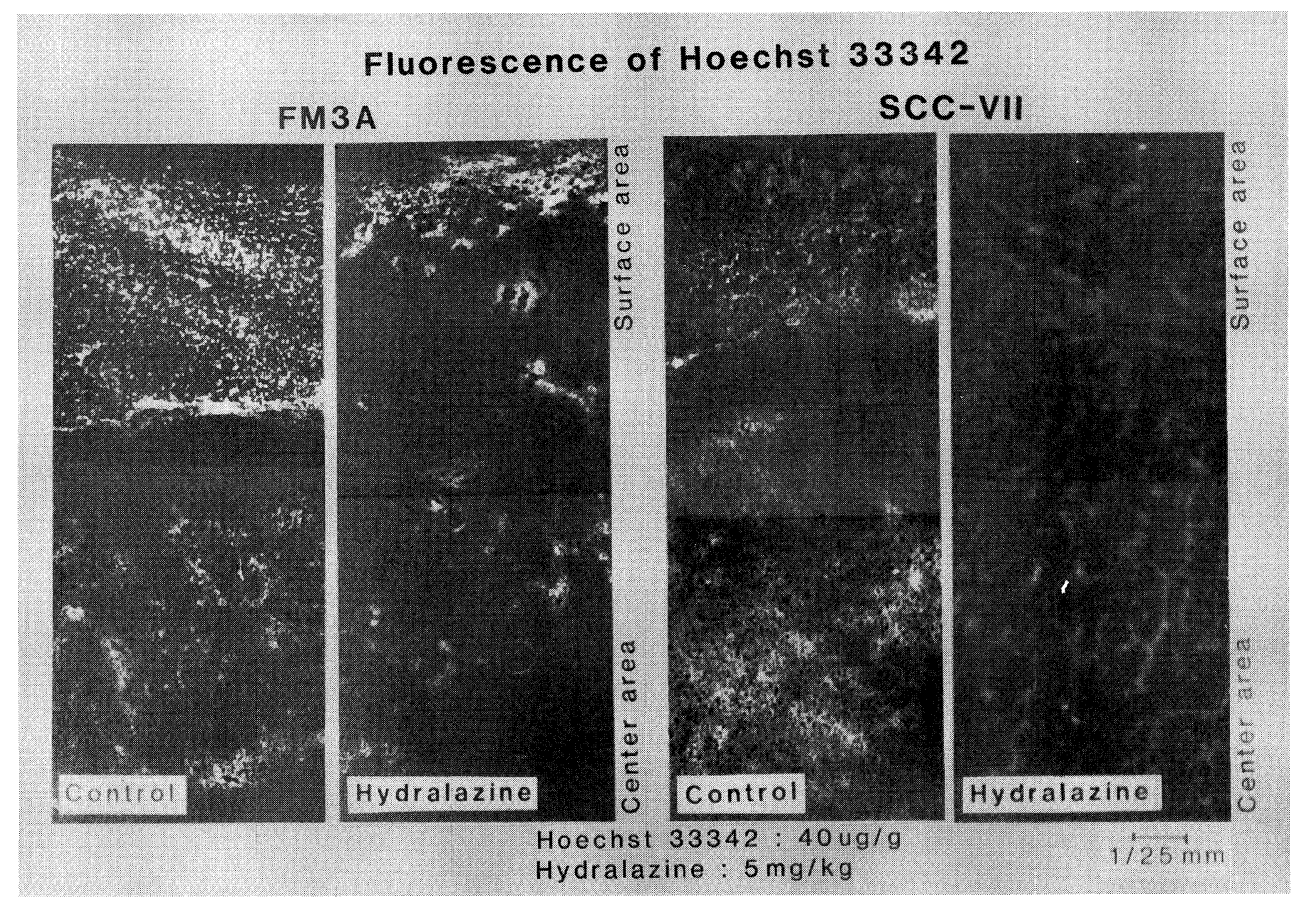

Fig. 2 Fluorescences showing blood distributions in FM3A and SCC-VII tumors after injection of hoechst-33342.

emission $=460 \mathrm{~nm}$, dose $=50 \mu \mathrm{g} / \mathrm{g}$ ), was infused into the tail vein and the animal was sacrificed by cervical dislocation 3 min later. Immediately thereafter, the tumor was surgically taken out and freezed at $-70^{\circ} \mathrm{C}$. The freezed tissue block was sliced into sections $10 \mu \mathrm{m}$ in thickness and the fluoresence distribution in the tissue was examined under a fluorescence microscope.

\section{Results}

Fig. 1 illustrates the normal tissue and tumor tissue of blood flow after HYD injection. The blood flow did not show the big changes in the intact musular tissue after HYD administration. In contrast, notable decreases in blood flow were recognized at all places within the tumor except for the necrosis area. An examination of the blood distribution using Hoechst-33342 revealed that the fluorescence intensity was significantly lowered in either the FM3A and the SCC-VII tumor after HYD injection. The blood flow and the oxygen tension were shown same tendency when compared with normal tissue and tumor tissue. This suggested that the local blood was markedly decreased by HYD administration (Fig. 2). This observation substantiated the HYD induced reduction in local blood flow. The tumor quadruping time was 4.5 days in the untreated FM3A group (control), 5.4 days in the HYD-treated FM3A group, 8.5 days in the heat treated FM3A, and 11.2 days in the HYD plus 


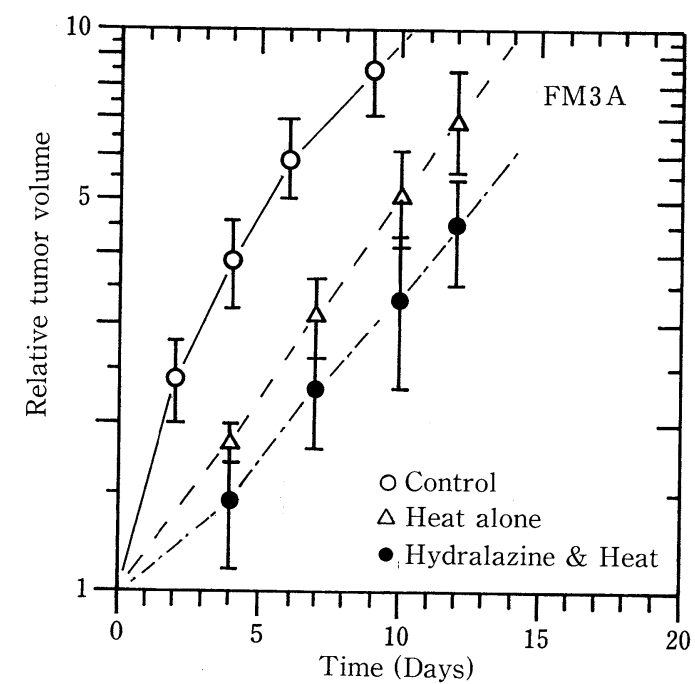

Fig. 3 FM3A tumor growth curves after various treatments. Each point represents a mean of determinations in 7 mice. Hyperthermia was given $20 \mathrm{~min}$ after injection of hydra lazine $(5 \mathrm{mg} / \mathrm{kg})$.

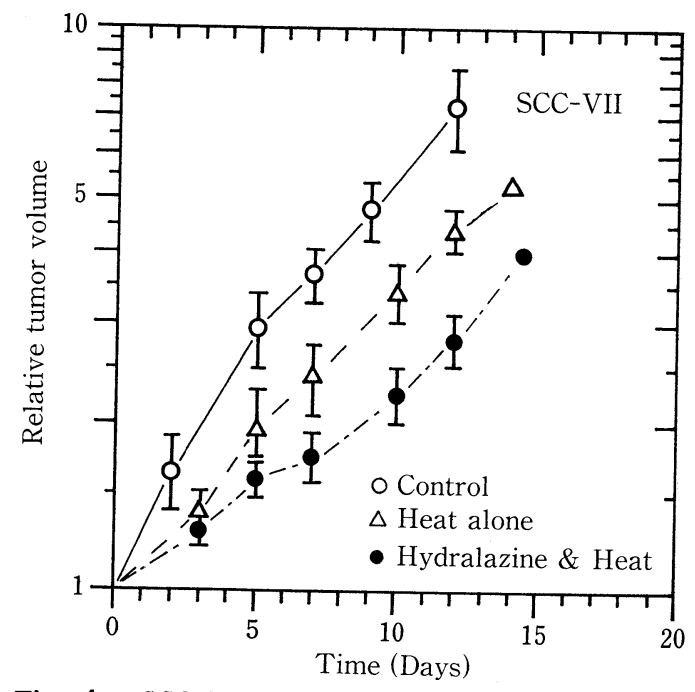

Fig. 4 SCC-VII tumor growth curves after various treatments, Each point represents a mean of determinations in 7 mice. Hyperthermia was given $20 \mathrm{~min}$ after injection of hydralazine $(5 \mathrm{mg} / \mathrm{kg})$.

heat treated FM3A group (Fig. 3).

Those in the respective SCC-VII groups were 7.7 days, 9.0 days, 11.1 days, and 14.0 days (Fig. 4). There was a correlation between the enhancement ratio and the degree of reduction in blood flow after HYD administration. To sum up, our findings suggest that HYD exerts a stealing effect, thereby decreasing blood flow in the tumor and promoting the carcino-static effect of hyperthermia. If the side effects of HYD can be diminished, its clinical application as an adjuvant to hyperthermia seems promising.

\section{N. Discussion}

Since it has a vasodilator action, it has long been utilized clinically as an antihypertensive drug. Pharmacokinetic studies have demonstrated that it acts on the enzyme system of the arteriolar smooth muscles, thereby dilating the vessels, and that it is catabolized by the mediation of $\mathrm{N}$-acetyltransferase ${ }^{7)}$. HYD induces vasodilution in normal tissues with smooth muscles, whereas it fails to do so in tumors which are deficient in smooth muscles. In normal tissues, therefore, the blood flow increases after HYD administration.

However, in tumor scarce of blood flow, nutrition and oxygen deliveries are deficient and the low blood flow area and hypoxic area increases, which results in a enhancement of the cytotoxicity of hyperthermia) ${ }^{6)}$ Besides, HYD is said to reduce tumor tissue blood flow selectively $^{8)}$. Some investigators used it in an attempt to enhance the efficacy of hyperther- 
mia for maligancies whose thermo-distribution was reduced ${ }^{9)}$. A reasonable inference from the foregoing is that HYD administration might be useful adjuvant to cancer therapy using a hyperthermia on condition that it is essential to find an optimum dose in consideration of the adverse drug reactions and aduquate counter measures that can taken.

\section{(Acknowledgments)}

We wish to express our sincere thanks to Professor Tustomu Kagiya (Kyoto University) for his helpful advice.

\section{Reforences}

1) Y. Tanaka, T. Hasegawa, T. Murata, et al. Effects of Hyperthermia Combined with Radiation on Normal and Tumor Microcirculation., FUNDAMENTALS OF CANCER THERAPY BY HYPERTHERMIA, RADIATION AND CHEMICALS., Edited by E. Kano., 95-109 Mag. Bros. Inc. Tokyo., (1982)

2) C. W. Song., Effect of Hyperthermia on Blood Flow and Microenvironment. Reviw. Cancer Research., Vol. 44, 4721s-4730s, (1984)

3) H. A. Eddy., Alterations in Tumor Microvasculature During Hyperthermía., Radiology, Vol. 137, 515-521, (1980)

4) H. I. Bicher, F. W. Hetzel, Sandhu, et al. Effects of Hyperthermia on Normal and Tumor Microenvironment., Radiology., Vol. 137., 523-530, (1980)

5) H. Yamamura, T. Matsuzawa, Tumor Regrowth after Irradiation. An experimental approach., Int. J. Radiat. Biol. Phys., Vol. 35., 201-219 (1979)

6) C. F. Babbs, D. P. Dewitt, W. D. Voorhees et al Theoretical Feasibility of Vasodilator-Enganced Local Tumor Heating., Eur. J. Cancer Clin., Vol. 18., No. 11, 1137-1146 (1982)

7) D. E. Drayer, M. M. Reidenberg., Clinical Consequences of Polymorphic Acetylation of Basic Drugs., Clin. Pharmacol. Thera., Vol. 22., 251-258, (1977)

8) T. Hasegawa, C. W. Song., Effect of Hydralazine on the Blood Flow in Tumor and Normal Tissues in Rats., Int. J. Radiat. Oncol. Biol. Phys., Vol. 20., No. 5, 1001-1007., (1991)

9) W. D. Voorhees III , C. F. Babbs., Hydralazine-Enhanced Selective Heating of Transmissible Venereal Tumor Im. plants in Dogs., Eur. J. Cancer Clin. Oncol., Vol. 18., 1027-1033., (1982) 